$r=-0.412$, respectively) and with hip fracture risk with and without FN-BMD ( $r$ $=-0.799, r=-0.412$, respectively). Major fracture risk with and without FN-BMD presented a moderate correlation with spine fractures $(r=0.350 ; r=0.397$, respectively).

No correlation was found between WHO threshold and spine fractures. No correlations were found between FN-BMD or fracture risk estimated by FRAX and disease manifestations, anti-Scl70 or ACA positivity, vitamin D insufficiency, smoking or GCT use.

Conclusion: In our cohort, low BMD was prevalent and had correlation with BMI. FRAX appears to be an useful instrument as it correlated with spine fractures, contrary to what was verified when we used the WHO threshold. Early monitoring of BMD and estimating fracture risk using FRAX appear to be useful tools for the prevention of fractures in this population.

Disclosure of Interests: : Salomé Garcia: None declared, Bruno Miguel Fernandes: None declared, Sara Ganhão: None declared, Maria Rato: None declared, Filipe Pinheiro: None declared, Georgina Terroso: None declared, Miguel Bernardes Speakers bureau: Abbvie, Amgen, Biogen, Eli-Lilly, GlaxoSmith-Kline, Pfizer, Janssen, Novartis, Lúcia Costa: None declared DOI: 10.1136/annrheumdis-2020-eular.2752

\section{SAT0321 CURRENT PATIENT REPORTED OUTCOMES (PROS) POORLY REFLECT CHANGES IN LUNG FUNCTION IN PATIENTS WITH SYSTEMIC SCLEROSIS}

F. Danzo ${ }^{1}$, K. Gjeloshi ${ }^{1}$, G. Abignano ${ }^{2,3,4}$, A. M. Dean ${ }^{3}$, F. Masini ${ }^{1}$, G. Cuomo ${ }^{1}$, F. Del Galdo ${ }^{3,4}$. 1 Università degli Studi della Campania, L. Vanvitelli, UOC Medicina Interna, Naples, Italy; ${ }^{2}$ Istituto Reumatologico Lucano (IReL), Azienda Ospedaliera Regionale San Carlo, Potenza, Italy; ${ }^{3}$ Leeds Institute of Rheumatic and Musculoskeletal Medicine, University of Leeds, Leeds, United Kingdom; ${ }^{4}$ NIHR Leeds Biomedical Research Centre, Leeds Teaching Hospital, Leeds, United Kingdom

Background: Lung involvement is very common is systemic sclerosis (SSc). Approximately one quarter of patients develops pulmonary problems within the first 3 years of diagnosis and still represents the leading cause of death in these patients. In a recent clinical trail, the reduction of FVC was not accompanied by a benefit with respect to health-related quality of life and patient-reported outcomes (PROs).

Objectives: To assess how the change in Pulmonary Function Test (PFTs) parameters correlates with the Patient Reported Outcomes (PROs) in an observational cohort of patients with Systemic Sclerosis (SSc).

Methods: We conducted a retrospective study of 330 clinic episodes from 121 unselected patients diagnosed with systemic sclerosis according to EULAR/ ACR 2013 criteria, in annual follow-up (for a total of 165 patients/year) with PFTs, Health Assessment Questionnaire Disability Index (HAQ-DI), Scleroderma Health Assessment Questionnaire (sHAQ), Modified Borg Dyspnea Scale (Borg) and Cochin Hand Function Score (CHFS). We assessed the correlation between the HAQ and the Visual Analogical Scale 1-7 at baseline (VAS1 pain, VAS2 disease severity, VAS3 arthritis activity, VAS4 intestinal problems, VAS5 dyspnea, VAS6 Raynaud's phenomenon, VAS7 digital ulcers). We evaluated the correlation of PFTs with PROs at every time period and the correlation between the change of PFTs parameters ( $\delta F V C, \delta D L C O)$ with the change of the PROs over a year of follow-up. Following analysis of distribution, Spearman or Pearson Test were used to determine correlation coefficients, as appropiate (Prism 7).

Results: The median disease duration was 5 years (IQR 3-10). The median of 12 months $\delta$ FVC\% and $\delta$ DLCO\% were 0 (IQR -5.81 to 3.28 ) and -2.439 (IQR -8.76 to 5.98$)$, respectively. The analysis evidenced a strong positive correlation between VAS1-7 and HAQ. We observed also significant correlation between FVC\%, DLCO \% and HAQ-DI ( $r=-0.355$ and -0.266 , respectively; $p<0.0001$ for both), Borg ( $r=-0.403$ and -0.379 , respectively; $p<0.0001)$ and CHFS $(r=$ -0.355 and -0.256 , respectively; $p<0.0001$ ). Nevertheless, in longitudinal setting there was no significant correlation between $\delta P R O s$ and changes lung function, as continuous variables, neither there was any significant PROs difference in patients that did or did not lose more than $10 \%$ of FVC and DLCO over a year of follow-up.

Conclusion: This analysis of a monocentric non-selected population evidenced that the current commonly used PROs in SSc while showing a good correlation with lung function are poorly sensitive to change or to reflect changes in lung function over 12 months. In this sense, prudent interpretation of the lack of correlation between FVC and patient-reported outcomes in studies of phase 3 is warranted.

References:

[1] Rahimi S., Nintedanib for systemic sclerosis-associated interstitial lung disease, Lancet Respiratory Medicine (2020)

Disclosure of Interests: None declared

DOI: 10.1136/annrheumdis-2020-eular.6494

\section{\begin{tabular}{|l|l}
\hline SAT0322 & PREVALENCE AND RISK FACTORS FOR LEFT
\end{tabular} VENTRICULAR DIASTOLIC DYSFUNCTION IN SYSTEMIC SCLEROSIS: RESULTS FROM RESCLE REGISTRY}

A. González ${ }^{1}$, J. L. Patier ${ }^{1}$, M. López-Rodríguez ${ }^{1}$, A. Guillén del Castillo², M. Rubio-Rivas ${ }^{3}$, A. Argibay ${ }^{4}$, B. Marí-Alfonso $0^{5}$, A. J. Chamorro ${ }^{6}$, A. B. Madroñero-Vuelta ${ }^{7}$, E. L. Callejas-Moraga ${ }^{5}$, C. González-Echávarri ${ }^{8}$, N. Ortego ${ }^{9}$, V. Fonollosa-Pla ${ }^{2}$, C. P. Simeón-Aznar ${ }^{2}$, O. B. O. R. I. Autoimmune Diseases Study Group (Geas) ${ }^{10}$ on behalf of RESCLE Investigators, Autoimmune Diseases Study Group (GEAS). ${ }^{1}$ Hospital Universitario Ramón y Cajal, Department of Internal Medicine, Madrid, Spain; ${ }^{2}$ Hospital Universitario Vall d'Hebron, Unit of Autoimmune Diseases, Department of Internal Medicine, Barcelona, Spain; ${ }^{3}$ Hospital Universitario de BellvitgeIDIBELL, Unit of Autoimmune Diseases, Department of Internal Medicine, L'Hospitalet de Llobregat, Barcelona, Spain; ${ }^{4}$ Complejo Hospitalario Universitario de Vigo, Unit of Systemic Autoimmune Diseases and Thrombosis. Department of Internal Medicine, Vigo, Pontevedra, Spain; ${ }^{5}$ Parc Taulí, Hospital Universitario, Department of Internal Medicine, Sabadell, Barcelona, Spain; ${ }^{6}$ Hospital Clínico Universitario de Salamanca-IBSAL Department of Internal Medicine, Salamanca, Spain; ${ }^{7}$ Hospital General San Jorge, Department of Internal Medicine, Huesca, Spain; ${ }^{8}$ Biocruces Bizkaia Health Research Institute, Hospital Universitario Cruces, University of the Basque Country, Autoimmune Diseases Research Unit, Department of Internal Medicine, Bilbao, Spain; ${ }^{9}$ Department of Medicine, Facultad de Medicina. Hospital Universitario San Cecilio, Inst Invest Biosanitaria Ibs Granada. Department of Internal Medicine, Unit of Systemic Autoimmune Diseases, Granada, Spain; ${ }^{10}$ Sociedad Española de Medicina Interna (SEMI), Madrid, Spain

Background: Left ventricular diastolic dysfunction (LVDD) is a very common finding in heart involvement in Systemic Sclerosis (SSc).

Objectives: To determine the prevalence, risk factors and mortality associated with LVDD in a cohort of patients with SSc.

Methods: A retrospective study was conducted with data from the multicentre Spanish Scleroderma Registry (RESCLE). A case-control study was performed to identify factors associated with LVDD.

Results: Out of 1517 cases of SSc, 319 (21\%) developed LVDD. Basal characteristics are shown in Table 1. In multivariate analysis, LVDD was associated to older age at diagnosis of SSc [54 vs 44 years, OR 1.05 (1,04-1.06)], presence of telangiectasia [ 67 vs $59 \%$, OR $1.42(1,88-1.08)$ ], and treatment with calcium channel blockers [50 vs. $45 \%$, OR $1.55(1.16-1.96)]$, and inversely correlated to treatment with ACE inhibitors [74 vs. $83 \%$, OR 0.59 (0.44-0.8)]. Mortality was increased in patients with LVDD (24 vs $17 \%$, OR 1.4, p = 0.01). Kaplan-Meier cumulative survival for the SSc cohort, according to the presence or absence of LVDD showed significant differences in 30 years from the first SSc symptom (59 vs. $70 \%, p=0.04$ )

Table 1.

\begin{tabular}{lcccc}
\hline Number of patients & 1517 & $319(21 \%)$ & $1198(79 \%)$ & $\mathbf{P}$ \\
\hline Limited SSc & $923(61 \%)$ & $201(63 \%)$ & $722(60 \%)$ & 0.438 \\
Diffuse SSc & $304(20 \%)$ & $51(16 \%)$ & $253(21 \%)$ & 0.041 \\
Sine Sclerodema SSc & $174(11 \%)$ & $47(15 \%)$ & $127(11 \%)$ & 0.048 \\
Age at disease onset (years) med \pm SD & $46.4 \pm 16.5$ & $54.1 \pm 15.9$ & $44.5 \pm 16.1$ & $<0.001$ \\
Arterial hypertensión & $491(33 \%)$ & $148(48 \%)$ & $343(30 \%)$ & $<0.001$ \\
Digital ulcers & $615(41 \%)$ & $108(34 \%)$ & $507(42 \%)$ & 0.006 \\
Telangiectasia & $918(61 \%)$ & $212(67 \%)$ & $706(59 \%)$ & 0.011 \\
Interstitial lung disease & $645(43 \%)$ & $153(48 \%)$ & $492(41 \%)$ & 0.021 \\
Pulmonary hypertension & $139(9.2 \%)$ & $34(11 \%)$ & $105(8.8 \%)$ & 0.325 \\
Capillaroscopy Slow pattern & $673(51 \%)$ & $130(46 \%)$ & $543(53 \%)$ & 0.038 \\
Centromere Antibodies & $679(49 \%)$ & $154(55 \%)$ & $525(48 \%)$ & 0.032 \\
\hline
\end{tabular}

Conclusion: In our cohort, LVDD is relative common in SSc patients, and it is associated with older age at diagnosis, treatment with calcium channel blockers and telangiectasia. ACE inhibitors could play a protective role against the development of LVDD. Mortality is higher in SSc patients with LVDD, and is more significative over time.

\section{References:}

[1] Tennøe $\mathrm{AH}$ et al. Left Ventricular Diastolic Dysfunction Predicts Mortality in Patients with Systemic Sclerosis. J Am Coll Cardiol. 2018;72:1804-13

Disclosure of Interests: Andrés González: None declared, Jose Luis Patier: None declared, Mónica López-Rodríguez: None declared, Alfredo Guillén del Castillo: None declared, Manuel Rubio-Rivas: None declared, Ana Argibay: None declared, Begoña Marí-Alfonso: None declared, Antonio-J Chamorro: None declared, Ana Belén Madroñero-Vuelta: None declared, Eduardo L. Callejas-Moraga: None declared, Cristina González-Echávarri: None declared, 